\title{
Topside measurements at Jicamarca during solar minimum
}

\author{
D. L. HyselI ${ }^{1}$, J. L. Chau ${ }^{2}$, and J. D. Huba ${ }^{3}$ \\ ${ }^{1}$ Earth and Atmospheric Sciences, Cornell University, Ithaca, NY, USA \\ ${ }^{2}$ Radio Observatorio de Jicamarca, Instituto Geofísico del Perú, Lima \\ ${ }^{3}$ Plasma Physics Division, Naval Research Laboratory, Washington, D.C., USA
}

Received: 12 September 2008 - Revised: 17 December 2008 - Accepted: 2 January 2009 - Published: 23 January 2009

\begin{abstract}
Long-pulse topside radar data acquired at Jicamarca and processed using full-profile analysis are compared to data processed using more conventional, range-gated approaches and with analytic and computational models. The salient features of the topside observations include a dramatic increase in the $T_{e} / T_{i}$ temperature ratio above the $F$ peak at dawn and a local minimum in the topside plasma temperature in the afternoon. The hydrogen ion fraction was found to exhibit hyperbolic tangent-shaped profiles that become shallow (gradually changing) above the $\mathrm{O}^{+}-\mathrm{H}^{+}$transition height during the day. The profile shapes are generally consistent with diffusive equilibrium, although shallowing to the point of changes in inflection can only be accounted for by taking the effects of $\boldsymbol{E} \times \boldsymbol{B}$ drifts and meridional winds into account. The SAMI2 model demonstrates this as well as the substantial effect that drifts and winds can have on topside temperatures. Significant quiet-time variability in the topside composition and temperatures may be due to variability in the mechanical forcing. Correlations between topside measurements and magnetometer data at Jicamarca support this hypothesis.
\end{abstract}

Keywords. Ionosphere (Equatorial ionosphere; Ion chemistry and composition) - Radio science (Instruments and techniques)

\section{Introduction}

A number of experiments aimed at measuring ionospheric plasma density, temperature, and composition were carried out at the Jicamarca Radio Observatory shortly after the facility became operational in the 1960s. Because of its low operating frequency $(49.92 \mathrm{MHz})$, Jicamarca can measure iono-

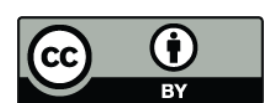

Correspondence to: D. L. Hysell (david.hysell@cornell.edu) spheric parameters at altitudes of several thousand kilometers without encountering limiting Debye length effects. However, because of the long correlation time of the echoes, particularly in the $\mathrm{O}^{+}$dominated part of the ionosphere where it is of the order of $1 \mathrm{~ms}$, and because of clutter from the equatorial electrojet and other sources, measuring ionospheric parameters becomes difficult at low altitudes. Jicamarca consequently employed a variety of pulsing schemes, including double pulse pairs and unmodulated pulses of different lengths (up to $5 \mathrm{~ms}$ ), to make the measurements. Interpulse periods had to be long enough to satisfy the duty cycle limitations of the transmitters, and incoherent integration times had to be correspondingly long to achieve reasonable statistical confidence. Nevertheless, the measurements could be made, owing to the high (4-5 MW) peak power capability of the observatory at that time and to the absence of interference and space debris. For a review of some of the early experiments at Jicamarca with many interesting anecdotes about how data were collected and processed, readers should consult Farley (1991).

Following the discovery of coherent scatter from fieldaligned plasma irregularities in the equatorial electrojet and the postsunset F-region (equatorial spread $F$ ), the emphasis at Jicamarca shifted to experiments with increased resolution, bandwidth, pulse rates, and numbers of receiver channels for interferometry (see for example Woodman and La Hoz, 1976; Farley et al., 1981). Incoherent scatter experiments designed to utilize Jicamarca's unique ability to measure highly accurate vector drifts by observing perpendicular to $\boldsymbol{B}$ were also emphasized (Woodman and Hagfors, 1969; Kudeki et al., 1999). Much of the original topside data were never published, and some of the details regarding their acquisition were lost. F-region density, temperature, and composition measurements were revived in the 1970s and 1980s with the reintroduction of the Faraday rotation/ double-pulse experiment (Pingree, 1990). However, the results persistently indicated unphysically low electron-ion temperature

Published by Copernicus Publications on behalf of the European Geosciences Union. 

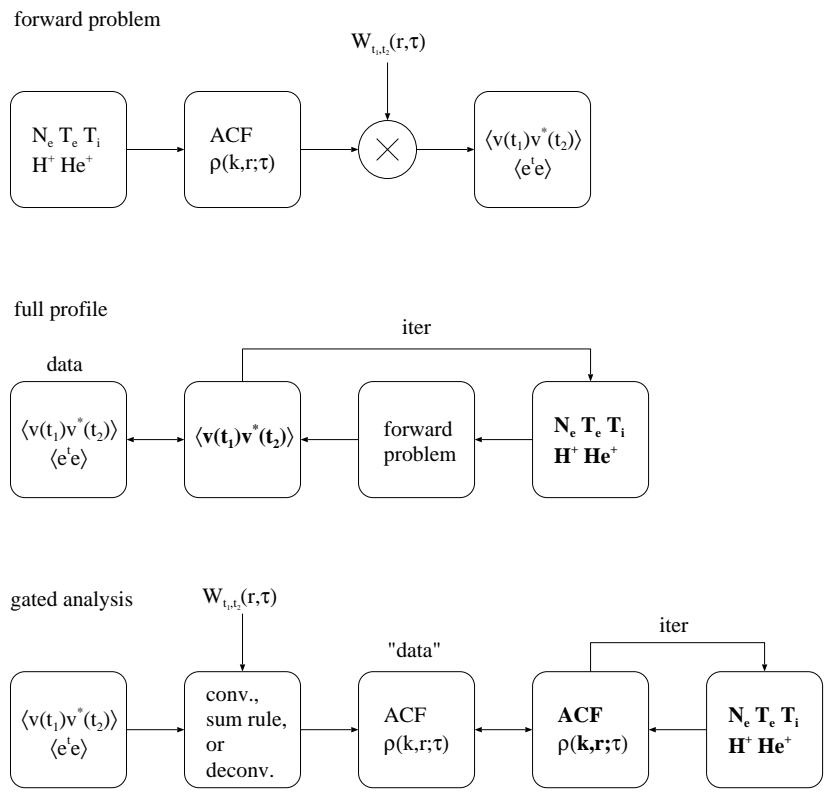

Fig. 1. Diagram comparing gated and full-profile analysis methods. Bold characters denote model parameter candidates (see text).

ratios $\left(T_{e} / T_{i}<1\right)$, and most of the data collected were never published.

The anomalous temperature ratio problem was resolved when Sulzer and Gonzalez (1999) analyzed the effects of electron Coulomb collisions on incoherent scatter theory at small magnetic aspect angles (see also Aponte et al., 2001; Woodman, 2004). Their work spurred an exhaustive investigation into the behavior of the incoherent scatter spectrum at small aspect angles, including collisional and magnetoionic effects, that continues to the present (Kudeki and Milla, 2006; Milla and Kudeki, 2006). Among other findings, this investigation showed how backscattered power profiles should be corrected for unequal electron and ion temperatures when calculating electron density profiles near the magnetic equator (Rodrigues et al., 2007). The correction is typically much smaller than the one required at large aspect angles. Today, most of the theoretical hurdles associated with density, temperature, and composition measurements at Jicamarca seem to have been cleared, although practical challenges remain. Improved instrumentation and data processing capabilities developed for other investigations are available for incoherent scatter experiments, and Jicamarca's peak power level is gradually returning to its historical peak.

Recently, Hysell et al. (2008) addressed several lingering experimental complications associated with long-pulse measurements in the topside. These include radar clutter from space debris, which is especially detrimental in long-pulse experiments and prevalent at the equator, and robust noise estimation. Most importantly, they implemented the fullprofile analysis proposed by Lehtinen (1986) and described in detail by Holt et al. (1992) and then Lehtinen et al. (1996) for resolving range-lag ambiguity, which is particularly important at Jicamarca given the long correlation time of the echoes. They presented the first topside results acquired at Jicamarca in several decades and compared the results with preliminary numerical models. In this manuscript, we apply the same techniques to begin establishing a baseline for equatorial composition and thermal structure. Where possible, we compare our results with results from more conventional radar data analysis methods and check for consistency. Most of the behavior observed can be inferred also from historical results, which can now be placed on firmer experimental foundations.

\section{Methodology}

Figure 1 illustrates the difference between conventional gated ISR data analysis and full-profile analysis. Incoherent scatter theory predicts the autocorrelation function $\rho(k, \tau)$ of thermal electron density fluctuations for an equilibrium, stationary, homogeneous plasma parametrized by number density $N_{e}$, electron temperature $T_{e}$, ion temperature $T_{i}$, hydrogen ion fraction $\mathrm{H}^{+}$, helium ion fraction $\mathrm{He}^{+}$, and other parameters including the magnetic field, line-of-sight drift, collisions, etc. (see for example Sheffield, 1975). The statistics of the signal received by the incoherent scatter radar are then determined by the same autocorrelation function, convolved with the two-dimensional range-lag ambiguity function $W_{t_{1}, t_{2}}(r, \tau)$ or instrument function for the experiment (see Lehtinen, 1986; Woodman, 1991; Lehtinen and Huuskonen, 1996; Nygrén, 1996). This function is determined by the characteristics of the transmitted pulse and the receiver filter and is independent of the characteristics of the radar target. Here, $r$ is the range to the scattering volume, $\tau$ is the time lag, and $t_{1}$ and $t_{2}$ are sample times separated by $\tau$. Parameters are permitted by incoherent scatter theory to vary significantly between different scattering volumes but only very gradually within a volume. The lag product estimator formed from the voltage samples, $\left\langle v\left(t_{1}\right) v^{*}\left(t_{2}\right)\right\rangle$, and the error covariance estimator that can be derived from it (Hysell, 2000), constitute the primary data from which inferences may be drawn. The covariance estimator is based on the data, and the radar pulse width and bandwidth must be set so that the estimator is sufficiently accurate for the analysis to proceed.

The first step in a traditional, range-gated analysis is to reconstruct distinct autocorrelation function estimates for each radar range gate from the lag product matrix. In the case of alternating code (Lehtinen, 1986) and coded longpulse (Sulzer, 1986) experiments, this can be accomplished through the explicit convolution of the received signal with the emitted waveform, an operation that can be performed efficiently in tandem with the calculation of the lag product matrix. Limitations of these methods include that 1 ) the zerolag estimator remains poorly range resolved and 2) the other lags suffer from significantly increased self clutter except in 
the limit of very low signal-to-noise (SNR) ratios. Whereas the signal-to-noise-plus-clutter ratio in a typical long-pulse experiment at Jicamarca is close to or greater than unity at all times and ranges of interest here in the first few lags, it is much less than unity in a typical 16-bit randomized alternating code experiment. Satisfactory results are only obtained given very long incoherent integration times, which are impractical to implement when conditions are changing rapidly.

A somewhat outmoded means of processing long-pulse ISR data involves reconstructing range-gated autocorrelation functions approximately using simple summation rules (Turunen, 1986). While this may produce satisfactory results in cases where the pulse length is short compared to ionospheric length scales of interest, the technique performs very poorly at Jicamarca near the $F$ peak of the ionosphere, particularly during the day when all topside parameters exhibit significant variations with altitude up to $1500 \mathrm{~km}$. Attempts to apply this simple technique produce enormous oscillations in fit temperatures and light ion fractions. Finally, range-resolved autocorrelation functions can be estimated from lag products matrices through direct numerical deconvolution (Virtanen et al., 2008; Nikoukar et al., 2008). The remaining steps in the gated analysis then involve estimating ionospheric parameters (state variables) gate-by-gate and iteratively using nonlinear least-squares methods.

In a full-profile analysis, lag product matrices and error covariances are calculated, but the intermediate range-gated autocorrelation functions are never reconstructed or fit. Instead, complete model parameter (state variable) profiles are generated directly and simultaneously. The forward model is used to calculate the corresponding candidate lag product matrices which are then compared with measurements. The chisquared model prediction error is minimized iteratively until satisfactory model-data agreement is reached. The model parameters themselves are typically discretized (20 values per profile in our case), but the forward model can be evaluated at arbitrary resolution using interpolation (cubic B-spline interpolation in our case - see De-Boor, 1978, for review).

However, the long-pulse analysis is generally mixed determined, meaning that there will be no model profiles that exactly reproduce the measured lag products but, rather, a large class of statistically admissible profiles that reproduce them adequately in terms of the chi-squared parameter. Furthermore, it is poorly conditioned, with many members of the class exhibiting large oscillations and irregular, non-physical behavior. Poor conditioning arises from a large range of singular values in the linearized version of the inverse problem and signals a tendency of the inversion to amplify statistical noise in the dataset (i.e. to "fit the noise"). Mixeddeterminedness and poor conditioning are inherent aspects of the long-pulse analysis problem rather than the analysis methodology. To overcome them, regularization may be used. For reviews of inverse methodology and the issues involved in regularization, see Menke (1984), Tarantola (1987), and Aster et al. (2005). Whereas range-lag ambi- guity can be overcome by pulse coding provided the added clutter is tolerable, rank deficiency and poor conditioning can be overcome by regularization provided there is sufficient a priori information upon which to draw. Note that it may be possible to improve the conditioning of the problem without adding clutter by modulating the long pulse, perhaps breaking it into two or more pulses of different lengths with intervening gaps. We recognize that possibility but do not explore it further here.

Regularization means reducing the solution space and excluding improbable solutions by incorporating a priori information about the model parameters. That the ionospheric state variables are non negative constitutes such a priori information. At equatorial latitudes, we furthermore expect the electron temperature to be greater than or equal to the ion temperature at all altitudes and the variations in model parameters to be gradual between adjacent ranges. An expedient way to implement regularization is to incorporate appropriate penalty functions into an augmented nonlinear leastsquares minimization of the usual chi-squared model prediction error.

Details regarding the analysis are given in Hysell et al. (2008) including the shape of the ambiguity functions in the forward model, the nonlinear least-squares methodology, the explicit form of the penalty functions, and the error propagation. Below $450 \mathrm{~km}$ altitude, long-pulse data are contaminated by clutter from various sources, and the experiment relies on data from a double-pulse Faraday rotation mode which is run concurrently. For computational expediency, the full-profile analysis includes a number of approximations with which we will dispense as computational limitations relax over time. Although it is possible to compute and utilize the full error covariance matrix, only the diagonal elements are used here. Among other effects, this likely implies underestimates in the derived parameter uncertainties. A thorough grid search is used at the start of the analysis to identify auspicious initial conditions for the analysis. The power profile, and the density profile from it, is calculated independently prior to the full-profile analysis. Since the ionospheric autocorrelation function is nearly flat near the zero lag, the relationship between the measured long-pulse power profile and the profile of power received from each radar range involves an essentially one-dimensional convolution. We perform a one-dimensional deconvolution using an augmented nonnegative least-squares approach for second-order Tikhonov regularization to recover the latter profile (Lawson and Hanson, 1987). This is normalized to the power profile from the double-pulse experiment and used thereafter in the forward model calculation.

Full-profile analysis comes with some rather severe limitations. It is not very robust and fails in the presence of even small amounts of interference, uncompensated noise, or undetected clutter from space debris or coherent echoes. Convergence fails in the absence of a suitable initial guess. It remains computationally intensive (although real-time 
processing has recently become practical). However, it appears to be the methodology most suitable for topside investigations at Jicamarca for the reasons outlined above.

\section{Observations}

The full-profile analysis has been applied to data acquired between 6-7 May 2008, and again between 8-13 July 2008, during the Coordinated Incoherent Scatter World Day period. The $10.7 \mathrm{~cm}$ solar flux was between 65-67 throughout both intervals. Geomagnetic activity was low at the start of the World Day period and increased to moderate at the end. In the May experiments, different pulsing modes were used serially in an effort to help validate the full-profile analysis. These included 1) a double-pulse Faraday rotation mode similar to the one described by Pingree (1990), 2) a mode combining the double pulse with randomized alternating coded pulses, similar to the one described by Hysell (2000), and 3) a mode combining the double pulse with long uncoded pulses similar to the one described by Hysell et al. (2008). All three modes are now available as common programs at Jicamarca. Full-profile is applied only to the long-pulse data acquired in the third of the modes.

Figure 2 shows data acquired on the first morning of the July 2008 World Day period. For this experiment, $0.1 \mathrm{~ms}$ double-pulse pairs are interleaved with a $1.6 \mathrm{~ms}$ long pulse, the overall sequence having a $40 \mathrm{~ms}$ interpulse period. Data are sampled using digital receivers and filters set to a $25 \mu \mathrm{s}$ impulse response time. Additional filtering (to $0.1 \mathrm{~ms}$ ), detection, and clutter removal using order statistics are performed computationally. Below (above) $450 \mathrm{~km}$ altitude, state parameters are derived from the double-pulse pair (long pulse data) only. The incoherent integration time is typically about 10 min.

The first column of Fig. 2 shows normalized autocorrelation functions derived from the double-pulse data. Vertical lines denote lags contaminated by clutter from the ground, the electrojet, and so-called 150-km echoes. The second column shows normalized lag products derived from the longpulse data. In both the first and second columns, data are represented by plotter symbols with error bars, and model fits by solid lines. The third column shows electron density. The green curve is derived from the short double pulses, which are transmitted on orthogonal circular polarizations, from Faraday rotation. This absolute electron density estimator is accurate but not robust and is used mainly to normalize the power profile corresponding to the double pulse zero lag density estimator, which is shown in black with error bars below $450 \mathrm{~km}$.

Above this altitude, electron density is estimated from the long-pulse zero lag. As mentioned above, a deconvolution scheme involving second-order Tikhonov regularization and a non-negative least-squares algorithm is used to compensate for the effect of the finite long-pulse length on the pro- file. We neglect the small effect that variations in the shape of the ACF has on the zero lag, making the deconvolution one-dimensional. The long-pulse plasma density estimator is normalized to the short-pulse estimator in the zone where the two curves overlap and shown in black with error bars above $450 \mathrm{~km}$ altitude.

The fourth column of Fig. 2 shows electron (ion) temperatures in black (red). Below $450 \mathrm{~km}$ altitude, these are obtained from conventional nonlinear least-squares fitting of the double-pulse ACFs. Equal electron and ion temperatures are assumed at night in the double-pulse analysis. Above this altitude, they are derived from the full-profile analysis of the long-pulse lag products. Similar comments hold for the light ion fraction estimates shown in the fifth column of Fig. 2, where black (blue) plotter symbols denote $\mathrm{H}^{+}\left(\mathrm{He}^{+}\right)$ ion fractions. Note that we never fit for helium ions in the double-pulse data. In the current analysis, we depart from our usual practice of also neglecting to fit for hydrogen ions below $450 \mathrm{~km}$. The very low solar flux in the summer of 2008 caused the $\mathrm{H}^{+}$fraction to be significant at times below $450 \mathrm{~km}$, particularly in the early morning, and so we include it as a fit parameter during the daytime here.

Figure 2 exemplifies a number of features common to $\mathrm{Ji}$ camarca daytime observations at solar minimum. The $\mathrm{H}^{+}$ fraction obeys a roughly hyperbolic tangent curve below the $\mathrm{O}^{+}-\mathrm{H}^{+}$transition height, which occurs near $550 \mathrm{~km}$ in this case. Above the transition height, the increase in $\mathrm{H}^{+}$fraction with altitude is more gradual than below. This feature is most obvious in the afternoon, when the curvature of the $\mathrm{H}^{+}$fraction shallows (varies more gradually with altitude), sometimes even changing inflection from convex to concave. The feature is not obviously correlated with secular variations in the temperature profiles. The scale height of the plasma number density also increases drastically above the transition height. $\mathrm{He}^{+}$appears only as a trace constituent but is usually also concentrated near the $\mathrm{O}^{+}-\mathrm{H}^{+}$transition height in the morning when it is most often detected.

The electron-ion temperature ratio is much greater than unity in the bottomside, where photoelectron production maximizes and ion-neutral cooling is efficient. The ratio is slightly greater than unity well into the topside, where the temperature increases gradually and nearly linearly in the $\mathrm{O}^{+}$-dominated part of the ionosphere. Statistical uncertainty makes it difficult to establish the asymptotic behavior of the temperature at the highest altitudes shown. Overall, parameter continuity across the $450-\mathrm{km}$ boundary altitude (which is not explicitly enforced) supports the efficacy of the fullprofile analysis.

Figure 3 shows a comparison of the full-profile analysis with conventional, range-gated analysis of randomized alternating code data, performed using data taken on 7 May 2008, explicitly for this purpose. The upper half of the figure shows the alternating code data, which have been incoherently integrated for an hour. The long-pulse data, shown below, were incoherently integrated for $10 \mathrm{~min}$. and processed 

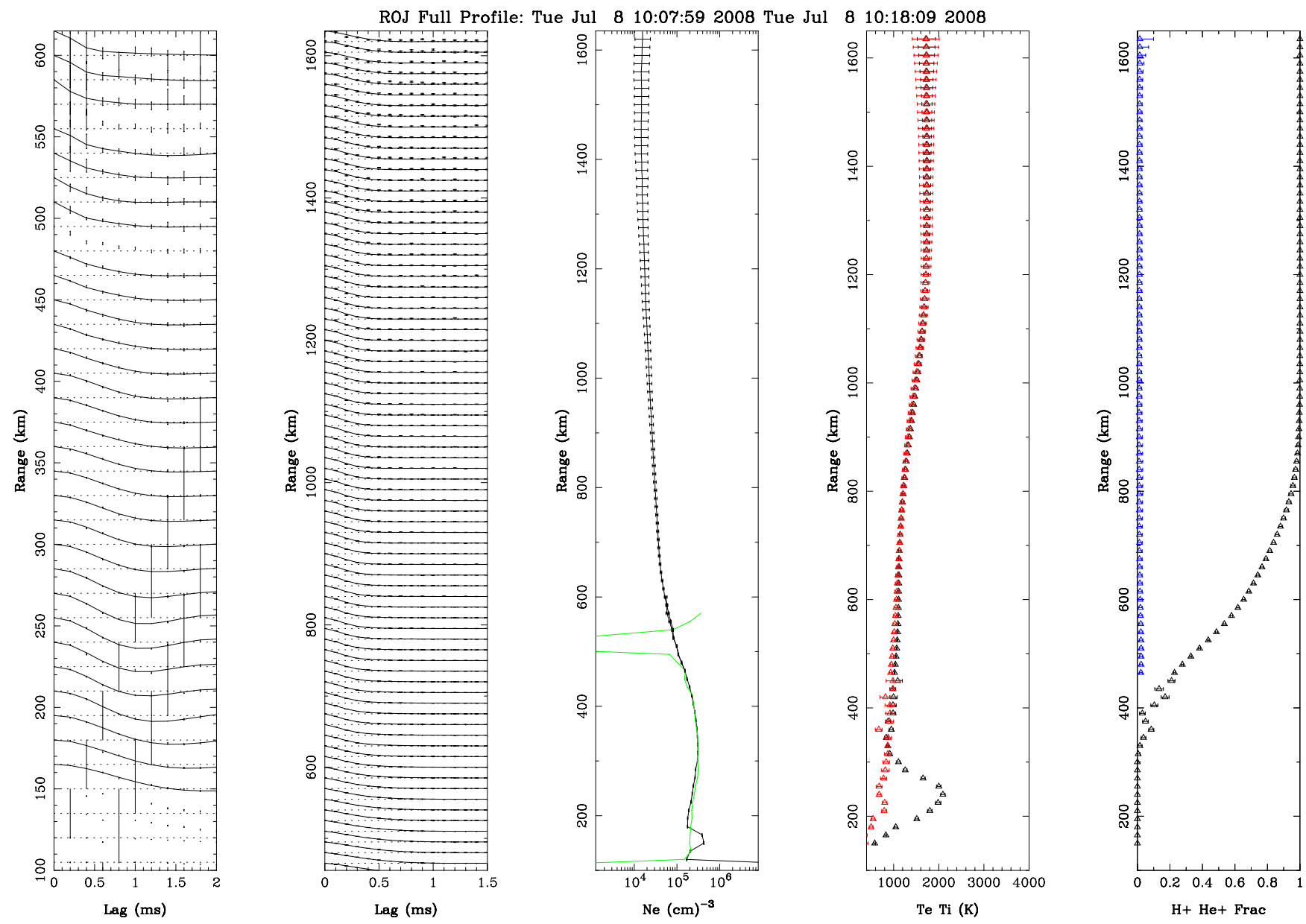

Fig. 2. Full-profile analysis results for the morning of 8 July 2008 from 10:08-10:18 LT. From left to right, the columns represent doublepulse autocorrelation functions, long-pulse lag products, plasma number density, plasma temperature (electrons $=$ black symbols, ions $=$ red symbols), and light-ion fraction $\left(\mathrm{H}^{+}=\right.$black, $\mathrm{He}^{+}=$blue $)$.

with full-profile analysis. The two datasets are nearly contiguous in time.

In the upper half of the figure, the first column shows double-pulse ACFs computed and analyzed in the manner already described. The second column shows range-gated autocorrelation functions computed from the $1.6 \mathrm{~ms}$, 16-bit randomized alternating code pulses. The third column shows plasma number density estimates, derived above $450 \mathrm{~km}$ by fitting the zero lag of the alternating code ACFs and normalizing to the double-pulse/ Faraday rotation data from below. The fourth column shows electron and ion temperatures, which are assumed to be equal above $450 \mathrm{~km}$. This assumption is required to avoid excessive jitter in the temperature fits, even given the long incoherent integration time used here. Finally, the fifth column shows $\mathrm{H}^{+}$fraction above $450 \mathrm{~km}$. Below $450 \mathrm{~km}$, the ionosphere is assumed to be entirely $\mathrm{O}^{+}$. Neglecting light ions in the double-pulse ACF analysis near and above the $F$ peak where significant $\mathrm{H}^{+}$ionization is possible often results in fit electron-ion temperature ratios below unity. When this occurs, a unity temperature ratio is enforced, and the given ACF is refit. That the electron and ion temperatures match at all altitudes above about $400 \mathrm{~km}$ in the upper half of Fig. 3 is a consequence of limitations in the fitting. Helium ions are neglected at all altitudes in this analysis.

In terms of the statistical confidence limits shown, agreement between the alternating coded and full-profile observations is satisfactory. Note that, being restricted to values less than unity, the $\mathrm{H}^{+}$fraction estimator becomes biased when it assumes values close to $100 \%$. It is significantly underestimated at high altitudes in the alternating code observations where statistical uncertainty is greatest. The corresponding temperatures are consequently overestimated at high altitudes. This accounts for the discrepancy between the two datasets starting at and above about $800 \mathrm{~km}$ altitude. Below this altitude, the features of the two datasets are in very good agreement. 

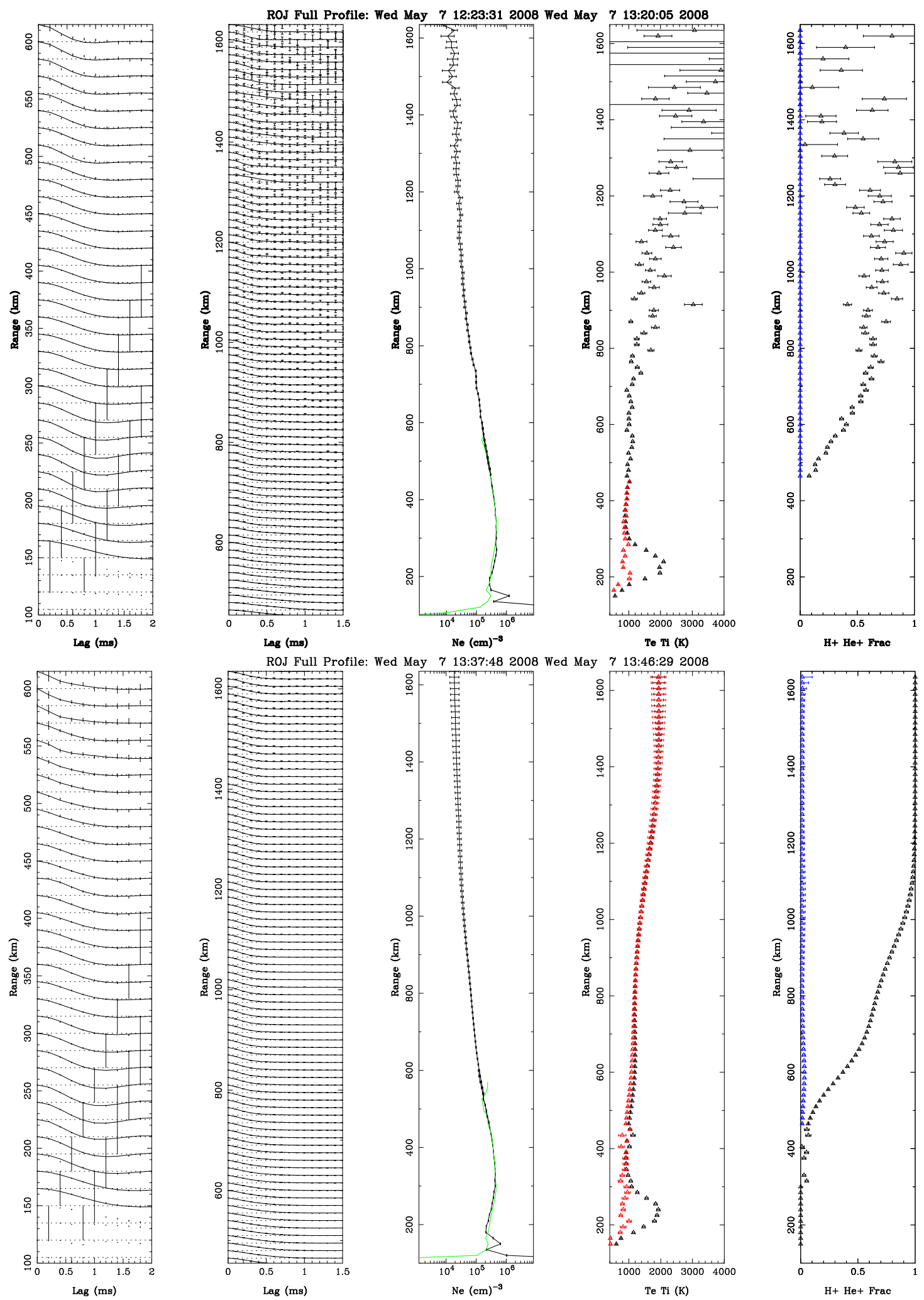

Fig. 3. Figures comparing alternating coded-pulse (upper half) and long-pulse/full-profile (lower half) results obtained on 7 May 2008. The incoherent integration times involved in the analysis were approximately $1 \mathrm{~h}$ and $10 \mathrm{~min} .$, respectively. 
Figure 4 shows ionospheric state parameters throughout the day of 7 May 2008, derived from the same kind of analysis that produced Fig. 2. From left to right, the columns represent the electron number density, electron temperature, ion temperature, and light ion fraction. In the last column, the curves concentrated toward the left are the helium ion fraction, which maximizes in the vicinity of the $\mathrm{O}^{+}-\mathrm{H}^{+}$transition height but never exceeds about 5\%.

The $\mathrm{O}^{+}-\mathrm{H}^{+}$transition height varies between about 500 $700 \mathrm{~km}$ altitude, reaching a maximum at about 14:00 LT. This is approximately the same time that the topside electron and ion temperatures reach their minimum values. We can surmise that the neutral temperature is greatest at this time, the neutral scale height the greatest, and conduction within and cooling of the plasma by the neutrals most efficient. Plasma $\boldsymbol{E} \times \boldsymbol{B}$ drifts which maximize at midday may also play a role, as we discuss in the analysis section below. Also at this time, the plasma scale height is minimized, and the topside plasma density exhibits a gradual decline with increasing altitude.

The topside plasma temperature is greatest near dawn (black curve) when conduction within the plasma and cooling by the neutral atmosphere is least efficient. The electronion temperature ratio is also drastically elevated for a few hours after sunrise in the topside, giving rise to local maximum in the electron temperature there. We associate the maximum with the heating by photoelectrons, able to penetrate into the topside of the ionosphere in the morning when plasma densities are still low.

The characteristics illustrated by Fig. 4 are similar to those exhibited in the observations shown by Hysell et al. (2008). Many of these characteristics were accurately reproduced by the NRL ionospheric model SAMI2 model (see Huba et al., 2000). SAMI2 reproduced very accurately the diurnal and altitude variation in plasma density and also hydrogen ion fraction. (Helium ion fraction was also accurately predicted, although at altitudes above the $\mathrm{O}^{+}-\mathrm{H}^{+}$transition height, whereas observations place it near or below the transition height.) Electron and ion temperatures were reproduced reasonably well, although there were some significant discrepancies. The electron-ion temperature ratio in the bottomside was underestimated. This may be a consequence of neglecting the heating of electrons through the quenching of an excited state of nitrogen $\left(\mathrm{N}\left({ }^{2} \mathrm{D}\right)\right)$ - see review by Aponte et al. (1999). More significantly, the model failed to reproduce the large electron-ion temperature ratio enhancement in the topside that occurs at sunrise. This is likely due to an oversimplified treatment of photoelectron transport, an effect that is now under investigation (W. E. Swartz, personal communication). Secondary ionization by photoelectrons is also neglected by the model, and this investigation may prompt a reinvestigation of the number density and composition predictions which appeared so successful.

Finally, Fig. 5 shows the first application of the full-profile analysis to a Jicamarca World Day dataset. The data span the interval from 8-13 July 2008. Only daytime data were collected in a mode suitable for this analysis. Note that the galactic center passed over the observatory between about 22:00-01:00 LT in early July. Because of this, the low plasma number density associated with the low solar flux, and an unseasonably high occurrence rate of plasma irregularities in the F-region associated with spread $F$, the incoherent scatter data acquired at night during this period suffer from a high degree of both clutter and statistical fluctuations. Moreover, the spread $F$ irregularities typically persisted for up to an hour after sunrise. The start times of the data blocks shown in Fig. 5 coincide with the cessation of spread $F$ irregularities and/or the emergence of significant F-region ionization.

In addition to incoherent scatter data, Fig. 5 also shows the difference in the $\mathrm{H}$ components of the magnetic field measured at Jicamarca $\left(0.8^{\circ} \mathrm{N}\right.$ dip angle) and Piura $\left(6.8^{\circ} \mathrm{N}\right.$ dip angle), $\sim 900 \mathrm{~km}$ to the north. This quantity is proportional to the equatorial electrojet current and may be regarded as a proxy for the zonal electric field in the ionosphere during the day (e.g. Anderson et al., 2004).

Figure 5 exhibits the same phenomenology as the dataset described by Hysell et al. (2008) while also illustrating a significant degree of day-to-day variability, particularly in the topside temperatures but also in composition. The main features include electron and ion temperature elevations at sunrise, electron-ion temperature ratio elevations at sunrise near the $F$ peak and throughout the day below the $F$ peak, a topside local temperature minimum at midday, a peak $\mathrm{O}^{+}-\mathrm{H}^{+}$ transition altitude at midday, a maximum in the $\mathrm{H}^{+}$topside scale height at midday, and the appearance of trace amounts of $\mathrm{He}^{+}$confined to a narrow layer $100-200 \mathrm{~km}$ thick near or below the $\mathrm{O}^{+}-\mathrm{H}^{+}$transition height during the day. The temperature and composition variability cannot be accounted for by variability in solar or geomagnetic forcing. However, variability in the temperatures and $\mathrm{H}^{+}$concentrations appear to be correlated with the magnetometer data, with the lowest topside temperatures and $\mathrm{H}^{+}$fractions occurring on the days when the electric field was strongest.

\section{Analysis}

The ionization and thermal structure of the equatorial topside ionosphere are controlled in large part by material and heat transport along magnetic flux tubes from sources and sinks residing primarily in the bottomside F-region. Photoelectron transport in particular seems to play a crucial role in topside electron heating at sunrise and may affect ionization rates through secondary ionization. Thermal conduction maintains topside electron and ion temperatures throughout the day. Light ions are produced either by charge exchange with $\mathrm{O}^{+}$in the case of $\mathrm{H}^{+}$or by direct ionization in the case of $\mathrm{He}^{+}$but are then transported upward along magnetic field lines by the ambipolar electric field in order that quasineutrality be maintained globally. Quantitative analysis of the 

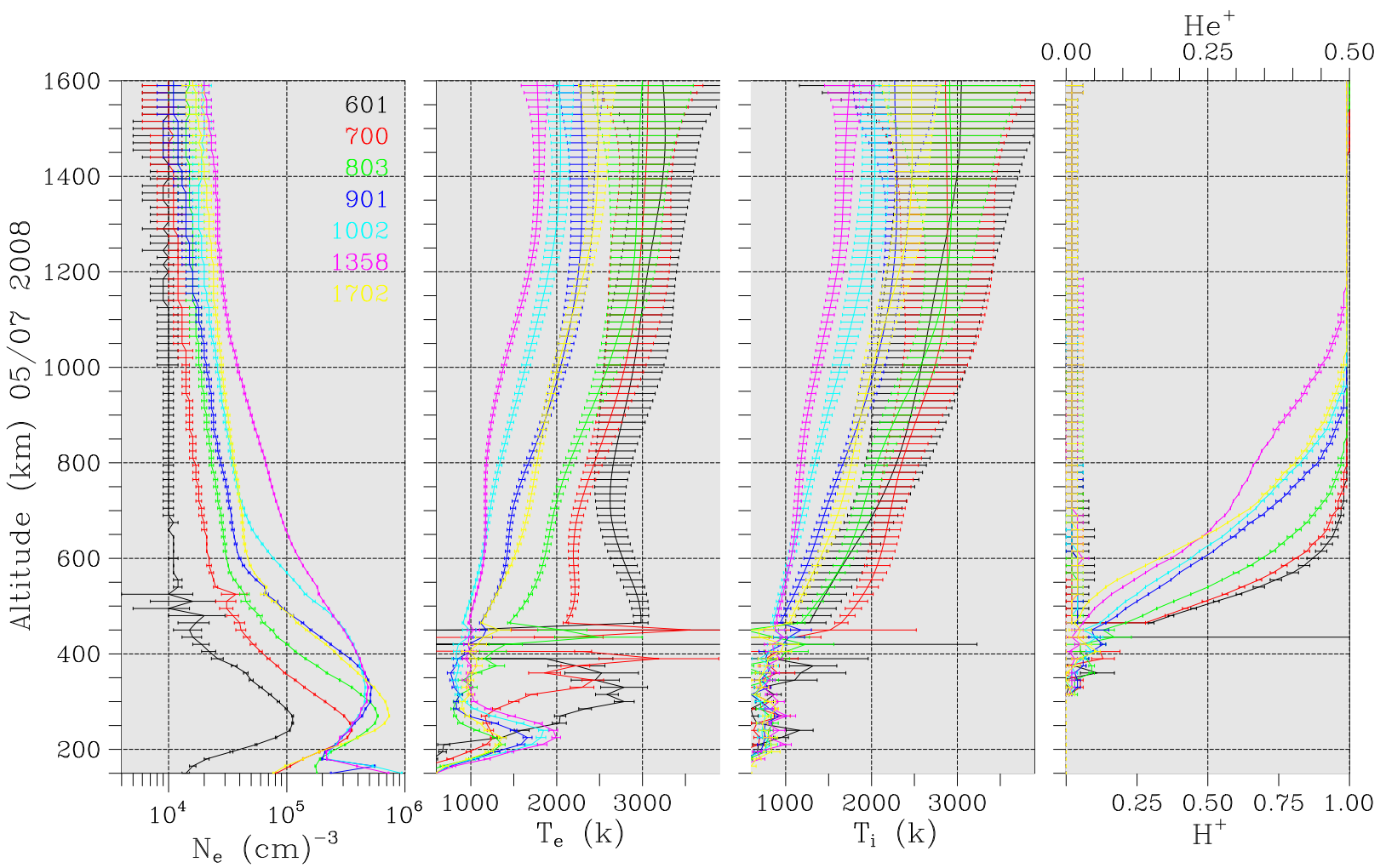

Fig. 4. Variations in ionospheric state parameters as a function of local time on 7 May 2008. The curves are based on 10-min. incoherent integrations. The four columns represent, from left to right, plasma number density, electron temperature, ion temperature, and light ion fraction. Long-pulse (double pulse) data are used for parameter estimation above (below) $450 \mathrm{~km}$ altitude. Data quality below $450 \mathrm{~km}$ altitude is poor in the early morning hours when plasma density is low.

observations presented here requires numerical tools capable of reproducing inherently nonlocal phenomena, and such tools are under development.

The diurnal behavior of the $\mathrm{O}^{+}-\mathrm{H}^{+}$transition height observed here is similar to what was found by González et al. (1992) who analyzed solar minimum AE-E satellite data from low latitudes. They also found that the $\mathrm{He}^{+}$concentration had a daytime maximum at equinox. At middle latitudes, meanwhile, the $\mathrm{He}^{+}$ion fraction has been shown to reach 10-20\% during solar minimum, but at night rather than during the day (González and Sulzer, 1996). Heelis et al. (1990) explained this behavior theoretically. At middle latitudes, helium ions produced by photoionization are pushed upward along magnetic flux tubes by the ambipolar electric field and concentrate near the $\mathrm{O}^{+}-\mathrm{H}^{+}$transition height. A reservoir of $\mathrm{He}^{+}$forms at altitudes where dissociative recombination is slow. Helium ions may even become the dominant species at some altitudes (Heelis et al., 1990; González et al., 2004). At the magnetic equator, however, helium ions are arrested at the flux tube apexes. The diurnal behavior of the $\boldsymbol{E} \times \boldsymbol{B}$ drift forces the flux tubes to low altitudes at night when helium ions are removed through dissociative recombination with molecular nitrogen. The $\mathrm{He}^{+}$fraction therefore peaks during the day and is limited to the amount of helium that can be produced in a single day.
An elementary but revealing analysis is one that predicts the light ion fractions as a function of altitude assuming diffusive equilibrium and relegating production and loss to altitudes below some lower boundary (e.g. González, 1994; González and Sulzer, 1996; González et al., 2004). Diffusive equilibrium is met when the force balance equations for the electrons and ions are satisfied:

$$
\begin{aligned}
0 & =-K \nabla\left(n_{j} T_{j}\right) / n_{j}+e E-m_{j} g \\
0 & =-K \nabla\left(n_{e} T_{e}\right) / n_{e}-e E-m_{e} g \\
n_{e} & =\sum_{j} n_{j}
\end{aligned}
$$

where $n_{j}$ is the number density of ion species $j, n_{e}$ is the electron number density, $T$ is the temperature, $E$ is the electric field, $m$ is the mass, $g$ represents gravity, and $K$ is the Boltzmann constant. Background electric fields and winds have been neglected here. Quasineutrality is enforced by Eq. (3). The equations are written in one dimension (the vertical), and the magnetic field is neglected. Since the magnetic field does not appear in the plasma Hamiltonian, it cannot affect the equilibrium distribution and the outcome of this analysis. We can solve this system of equations for a multipleion plasma for the number density profiles and the fractional 

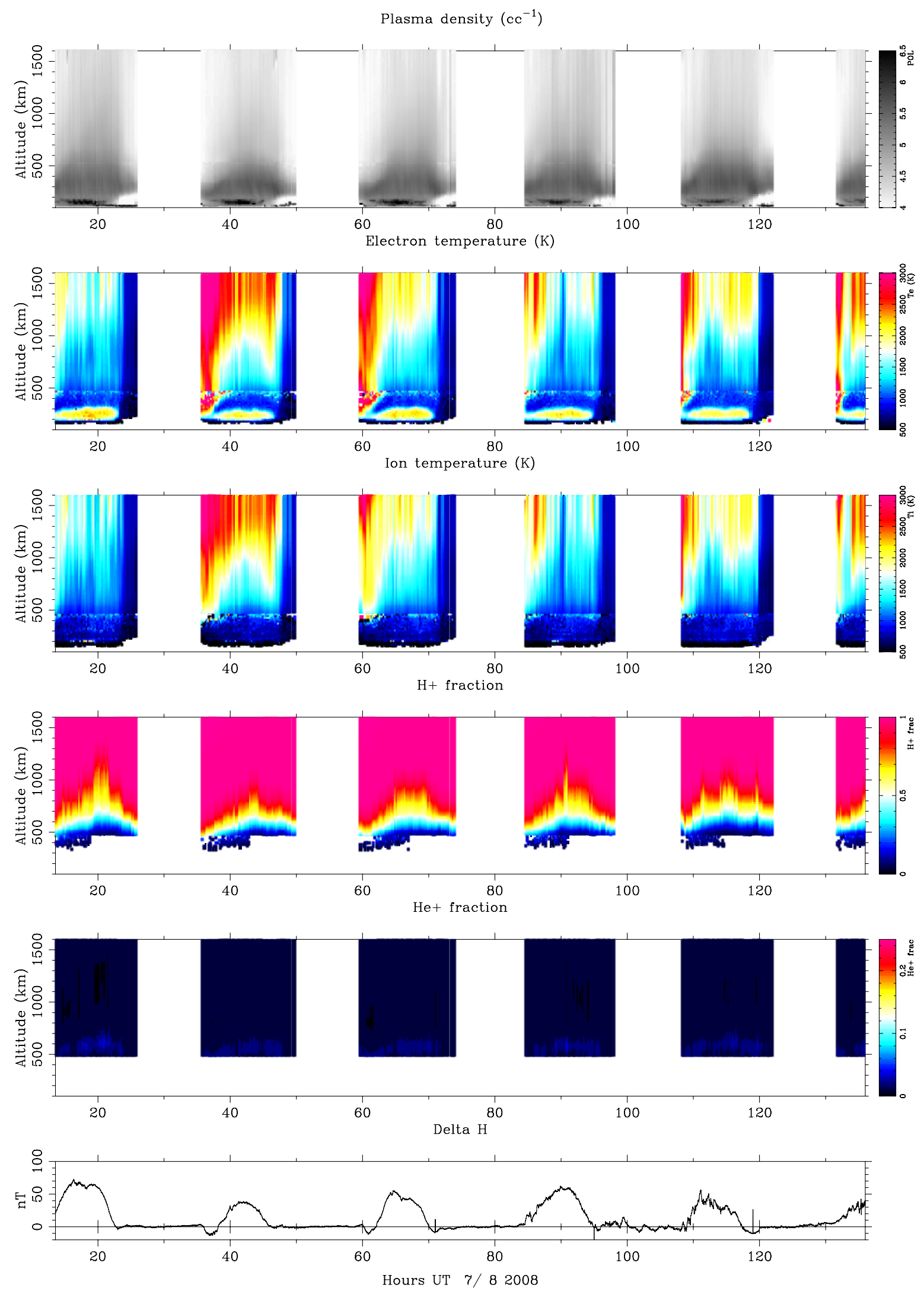

Fig. 5. Full-profile results for the July 2008 World Day period. From top to bottom, the rows depict plasma number density, electron temperature, ion temperature, hydrogen ion fraction, helium ion fraction, and the $\mathrm{H}$ component of the magnetic field due to the electrojet. 


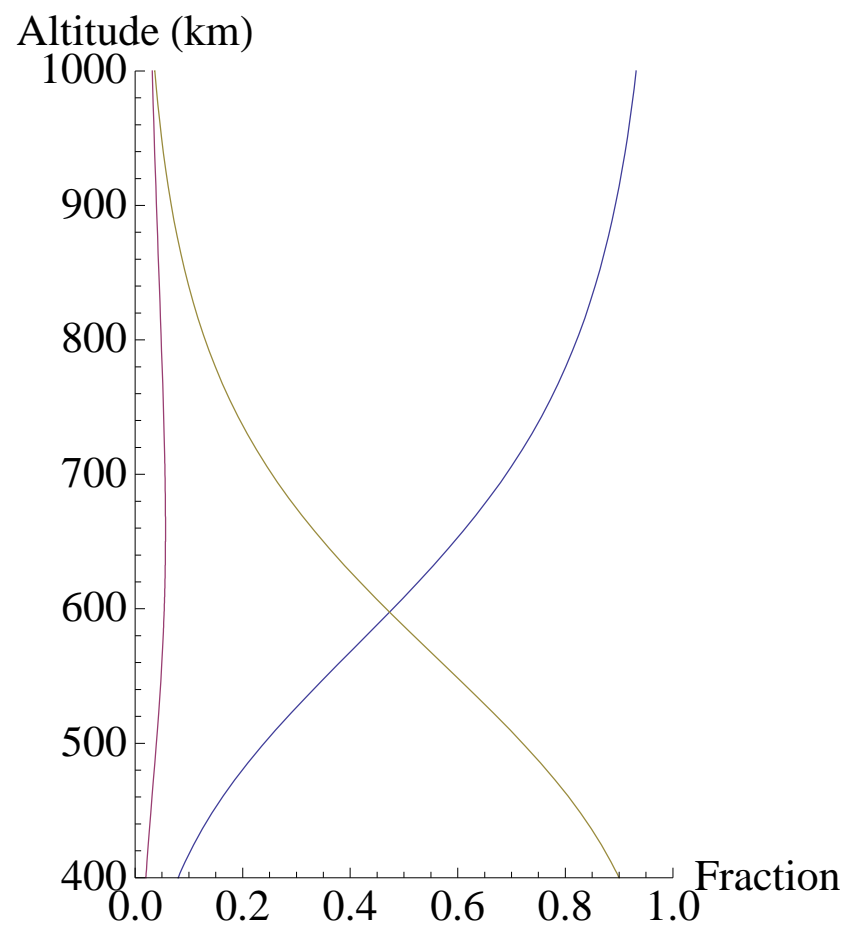

Fig. 6. Plot of Eq. (5) for a 3-ion plasma versus altitude above a $400 \mathrm{~km}$ reference altitude where the relative abundances are fixed as $\mathrm{O}^{+}: \mathrm{H}^{+}: \mathrm{He}^{+}=0.9: 0.08: 0.02$. The ion species share the same temperature, which increases with altitude (see text).

composition profiles:

$$
\begin{aligned}
n_{j}(z) n_{e}(z) & =n_{\circ j} n_{\circ e}\left(T_{\circ j} T_{\circ e} / T_{j} T_{e}\right) e^{-\int_{\circ}^{z} d z / H_{j}} \\
\frac{n_{j}(z)}{n_{e}(z)} & =\frac{n_{\circ j}\left(T_{\circ j} / T_{j}\right) e^{-\int_{\circ}^{z} d z / H_{j}}}{\sum_{i} n_{\circ i}\left(T_{\circ i} / T_{i}\right) e^{-\int_{\circ}^{z} d z / H_{i}}} \\
H_{j} & \equiv \frac{K T_{j}(z)}{m_{j} g(z)}
\end{aligned}
$$

where the temperatures for the plasma constituents are allowed to differ and to vary with altitude, along with the gravitational constant. Here, terms with zero subscripts represent boundary conditions specified at some lower altitude boundary where diffusive equilibrium holds. Note that the electron temperature has no effect on the fractional ion composition given by Eq. (5), although it obviously affects the absolute abundances.

Figure 6 shows Eq. (5) evaluated for a three-ion plasma: oxygen (green), hydrogen (blue), and helium (red) ions. In the computation, the ions have a common temperature which increases linearly from $800 \mathrm{~K}$ at the bottom of the figure to $1400 \mathrm{~K}$ at the top. The altitudes shown are above a $400 \mathrm{~km}$ reference altitude where transport is provisionally assumed to dominate photochemistry. The overall shape of the $\mathrm{H}^{+}$ fraction curve, including the gradual shallowing of the curve with increasing altitude, is similar to that found in the rightmost panel of Fig. 2. That the $\mathrm{He}^{+}$fraction maximizes near the $\mathrm{O}^{+}-\mathrm{H}^{+}$transition height also resembles observations, although the helium ions sometimes observed at Jicamarca tend to be concentrated in layers only about $100-200 \mathrm{~km}$ thick.

In fact, diffusive equilibrium is generally a poor model for the equatorial ionosphere below the protonosphere. Light ion photochemistry competes with transport at altitudes above $400 \mathrm{~km}$, and horizontal winds and the $\boldsymbol{E} \times \boldsymbol{B}$ drift motion of the equatorial flux tubes also force departures from diffusive equilibrium. Once disturbed, equilibrium is only restored very gradually given the slowness of cross-field material and heat transport.

The agreement of Figs. 6 and 2 is partly fortuitous and due to the facts that 1) the $\mathrm{H}^{+}$concentration curve predicted by photochemical equilibrium is similar to that predicted by diffusive equilibrium and 2) that $\mathrm{He}^{+}$is taken to be a minor constituent. The charge-exchange reaction controlling hydrogen ion photochemistry is $\mathrm{O}^{+}+\mathrm{H} \leftrightarrow \mathrm{H}^{+}+\mathrm{O}$. The forward and reverse reactions proceed at somewhat different, temperaturedependent rates, but for typical topside conditions, the rate balance condition leads to the following expression for the hydrogen ion fraction (see Schunk and Nagy, 2000, for example):

$$
\frac{n\left(\mathrm{H}^{+}\right)}{n\left(\mathrm{H}^{+}\right)+n\left(\mathrm{O}^{+}\right)}=\frac{\alpha n(\mathrm{H})}{\alpha n(\mathrm{H})+n(\mathrm{O})}
$$

where $\alpha=1.13$ and where helium ions are neglected. With neutral hydrogen and oxygen density profiles being governed by the scale height expressions in Eq. (6), Eq. (7) assumes a form very similar to Eq. (5), only with neutral temperature replacing ion temperature and with a small correction due to the $\alpha$ factor.

We can assess the impact of $\boldsymbol{E} \times \boldsymbol{B}$ drifts and horizontal (meridional) winds on the topside by comparing observations with additional SAMI2 model runs. Figure 7 shows ion fraction and ion temperature profiles computed with SAMI2 for June solstice, solar-minimum Jicamarca conditions. The upper-left panel reflects results assuming vertical plasma drifts specified by the Fejer-Scherliess empirical model (Scherliess and Fejer, 1999) and meridional winds prescribed by the HWM wind model (Hedin, 1991). Although run for a slightly later local time, the figure reproduces well the hydrogen ion fraction and ion temperature profiles seen in the long-pulse data in the lower half of Fig. 3. The predicted $\mathrm{H}^{+}$fraction shallows above the $\mathrm{O}^{+}-\mathrm{H}^{+}$transition height and assumes a concave curvature, even where the temperature profile is essentially flat, just as it often appears to in Jicamarca afternoon observations

We contrast this with the behavior predicted in the upperright panel of Fig. 7, for which the imposed Fejer-Scherliess vertical drifts were scaled by a factor of 0.25 . In this case, the hydrogen fraction profile remains nearly symmetric above and below the $\mathrm{O}^{+}-\mathrm{H}^{+}$transition height (which itself is little changed between the two runs), despite the fact that the ion temperature now increases drastically near and above the 
transition. Evidently, the equatorial $\boldsymbol{E} \times \boldsymbol{B}$ drift time history has a significant effect on the shape of the hydrogen ion fraction profile in the topside. Even more significant is the effect it appears to have on the topside temperature profile. The topside temperatures depicted in the left and right panels of Fig. 7 are very different, with higher temperatures accompanying slower daytime upward drifts. The sensitivity of the former to the latter appears to be rather severe.

The lower two panels of Fig. 7 represent simulation results where the neutral winds are set to zero. Fejer-Scherliess vertical drifts were used in the run shown in the lower-left panel, and drifts scaled by a factor of 0.25 were used in the lower right. The effects of suppressing the winds are to 1) suppress the topside temperatures, 2) depress the $\mathrm{O}^{+}-\mathrm{H}^{+}$transition height, 3) greatly reduce the $\mathrm{O}^{+}$fraction above the transition height, and 4) greatly reduce the peak height and intensify the $\mathrm{He}^{+}$layer. The overall behavior of the curves in the lowerright panel of Fig. 7, where the $\boldsymbol{E} \times \boldsymbol{B}$ drifts and meridional winds have been suppressed, is that predicted for diffusive equilibrium.

The simulation results predict much of the behavior seen in Fig. 5, where the days with the lowest topside temperatures and $\mathrm{H}+$ fractions are the days with the largest zonal electric fields, as inferred from magnetometer data during the day. In that regard, the simulations provide an explanation, in large part, for the high degree of quiet-time day-to-day variability observed. Some of the hourly variability in the topside may also be a time-integrated response to variations in electric field forcing, although further observation and analysis will be required to demonstrate this. First attempts to obtain accurate electric field estimates during the day and at night from long-pulse radar date are currently being made.

Meridional neutral winds also likely contribute to variability in the ion temperature and composition. Measuring meridional winds in the thermosphere from incoherent scatter will always be difficult at Jicamarca, although seasonallyaveraged profiles may eventually be obtainable. A more auspicious strategy for identifying and quantifying the effects of thermospheric winds in the topside would be to incorporate data from the Neutral Wind Meter instrument on the Air Force C/NOFS satellite (when available).

Finally, Fig. 7 suggests that the helium ion fraction is also strongly influenced by the zonal electric field and meridional winds. The simulation suggests that significant $\mathrm{He}^{+}$layers may only be observable when both are relatively small, and then only at altitudes near the $\mathrm{O}^{+}-\mathrm{H}^{+}$transition height. The helium ion fraction remained very small $(\lesssim 5 \%)$ throughout the observations described here but reached $10 \%$ in sporadic daytime layers near the transition height in the 2006 Jicamarca data examined by Hysell et al. (2008). Future studies will be targeted at correlating layer appearances such as those with external mechanical forcing.
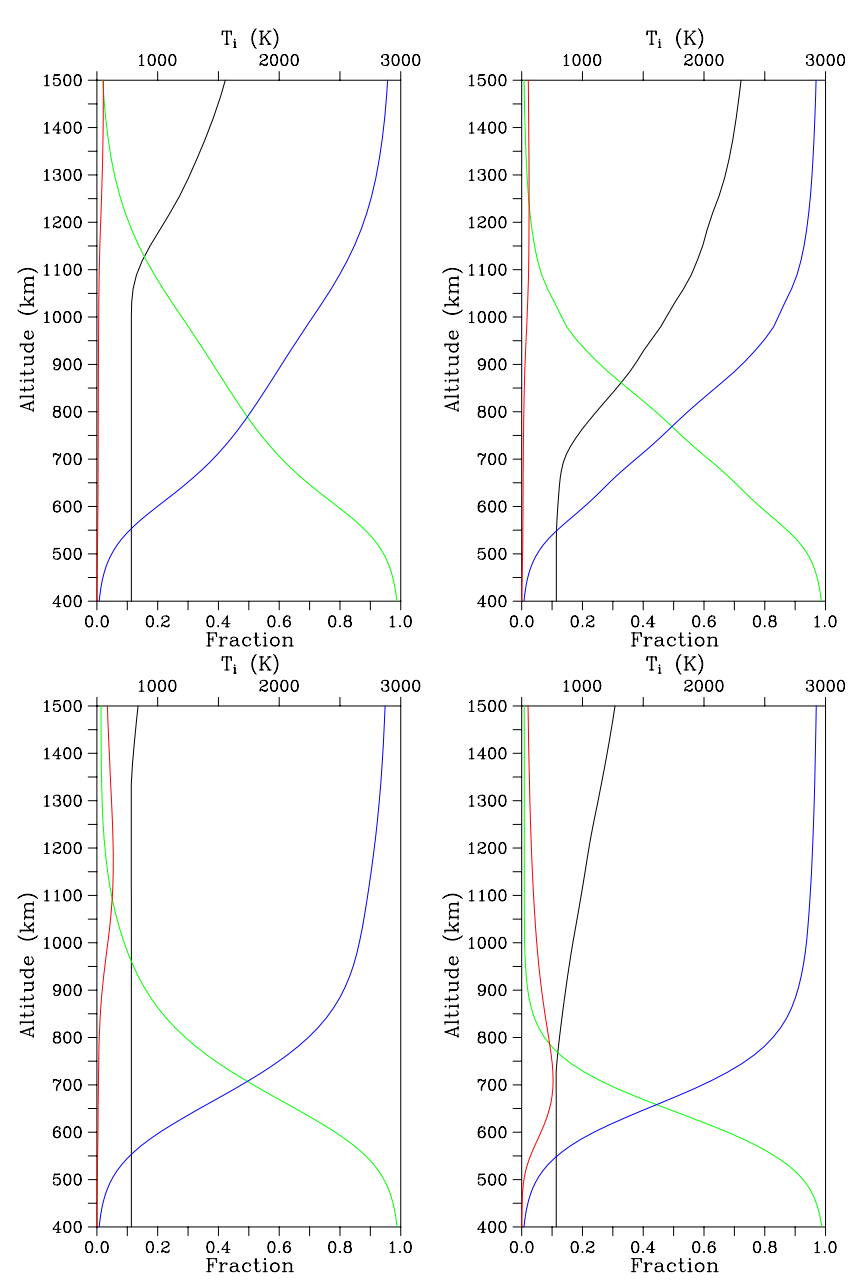

Fig. 7. SAMI 2 simulation runs for Jicamarca June solstice, solar minimum conditions at 15 LT. Oxygen, hydrogen, and helium ion fractions are shown in green, blue, and red as in the previous figure. The black curves show ion temperature. The left panels reflect simulations based on Fejer-Scherliess empirical vertical drifts. For the simulations depicted in the right panels, the vertical drifts were reduced by a factor of 4 . The upper panels reflect simulations incorporating HWM meridional neutral winds. Winds were set to zero for the simulations in the lower panels.

\section{Summary}

Long-pulse topside data acquired at Jicamarca and processed using a full-profile analysis approach have been compared to data analyzed using more conventional approaches, to a simple composition model based on diffusive equilibrium, and to predictions from the SAMI2 ionospheric model. Hydrogen ion fraction appears to be well approximated by the diffusive equilibrium model at times, although a model based on photochemical equilibrium gives similar results. The SAMI2 model shows that extreme shallowing of the $\mathrm{H}^{+}$fraction profile above the $\mathrm{O}^{+}-\mathrm{H}^{+}$transition height can be caused by 
upward daytime vertical $\boldsymbol{E} \times \boldsymbol{B}$ drifts when meridional winds are also present.

The same drifts and meridional winds can also have dramatic effects on the topside ion temperature profile. This seems to be a likely explanation for the strong quiet-time variability observed in the topside temperature profiles. While magnetometer data support the idea that topside temperature and light ion composition are very sensitive to the zonal electric field at solar minimum, definitive experimental verification requires improvements in Jicamarca's ability to measure vertical drifts in oblique antenna pointing experiments directly. Thermospheric neutral wind measurements are also required but present significant technical challenges for radar measurements. Similar remarks hold for variability in $\mathrm{He}^{+}$concentration, which is only observed sporadically and which is predicted to be suppressed by external mechanical forcing.

A common feature of the topside observations is the substantial increase of the electron-ion temperature ratio at sunrise each day at altitudes above the $F$ peak. This feature does not appear to be captured by the SAMI2 model, due most likely to an oversimplified treatment of photoelectron transport. Research is presently focused on improving this aspect of the model. Not only the temperature but also the density and composition could be affected, since secondary ionization by photoelectrons may be important.

Acknowledgements. We appreciate helpful advice from C. La Hoz, W. E. Swartz, and D. T. Farley. This research has been funded, in part, by ONR (JDH). The Jicamarca Radio Observatory is a facility of the Instituto Geofisíco del Perú operated with support from NSF cooperative agreement ATM-0432565 through Cornell University. We appreciate the help of the Jicamarca staff, particularly that of L. Condori.

Topical Editor K. Kauristie thanks M. Sulzer and another anonymous referee for their help in evaluating this paper.

\section{References}

Anderson, D. N., Anghel, A., Chau, J., and Veliz, O.: Daytime vertical $\mathrm{E} \times \mathrm{B}$ drift velocities inferred from ground based magnetometer observations at low latitudes, Space Weather, 2, S11001, doi:10.1029/2004SW000,095, 2004.

Aponte, N., Swartz, W. E., and Farley, D. T.: Electron energy balance in the $F$ region above Jicamarca, J. Geophys. Res., 104(A5), 10041-10049, 1999.

Aponte, N., Sulzer, M. P., and Gonzales, S. A.: Correction of the Jicamarca electron-ion temperature ratio problem: verifying the effect of electron Coulomb collisions on the incoherent scatter spectrum, J. Geophys. Res., 106(A11), 24785-24794, 2001.

Aster, R. C., Borchers, B., and Thurber, C. H.: Parameter Estimation and Inverse Problems, Elsevier, New York, 2005.

De-Boor, C.: A Practical Guide to Splines, Springer Verlag, New York, 1978.

Farley, D. T.: Early incoherent scatter observations at Jicamarca, J. Atmos. Terr. Phys., 53, 665-675, 1991.
Farley, D. T., Ierkic, H. M., and Fejer, B. G.: Radar interferometry: A new technique for studying plasma turbulence in the ionosphere, J. Geophys. Res., 86, 1467-1472, 1981.

González, S. A.: Radar, satellite, and modelling studies of the low latitude protonosphere, PhD thesis, Utah State University, 1994.

González, S. A. and Sulzer, M. P.: Detection of $\mathrm{He}^{+}$layering in the topside ionosphere over Arecibo during equinox solar minimum conditions, Geophys. Res. Lett., 23, 2509-2512, 1996.

González, S. A., Fejer, B. G., Heelis, R. A., and Hanson, W. B.: Ion composition of the topside equatorial ionosphere during solar minimum, J. Geophys. Res., 97, 4299-4303, 1992.

González, S. A., Sulzer, M. P., Nicolls, M. J., and Kerr, R. B.: Solar cycle variability of nighttime topside helium ion concentration over Arecibo, J. Geophys. Res., 109, A07302, doi:10.1029/2003JA010100, 2004.

Hedin, A. E.: Extension of the MSIS theromspheric model into the lower and middle atmosphere, J. Geophys. Res., 96, 1159-1172, 1991.

Heelis, R. A., Hanson, W. B., and Bailey, G. J.: Distributions of $\mathrm{He}^{+}$at middle and equatorial latitudes during solar maximum, $\mathrm{J}$. Geophys. Res., 95, 10313-10320, 1990.

Holt, J. M., Rhoda, D. A., Tetenbaum, D., and van Eyken, A. P.: Optimal analysis of incoherent scatter radar data, Radio Sci., 27, 435-447, 1992.

Huba, J. D., Joyce, G., and Fedder, J. A.: Sami2 is another model of the ionosphere (SAMI2): A new low-latitude ionospheric model, J. Geophys. Res., 105, 23035-23054, 2000.

Hysell, D. L.: Incoherent scatter experiments at Jicamarca using alternating codes, Radio Sci., 35, 1425-1436, 2000.

Hysell, D. L., Rodrigues, F. S., Chau, J. L., and Huba, J. D.: Full profile incoherent scatter analysis at Jicamarca, Ann. Geophys., 26, 59-75, 2008, http://www.ann-geophys.net/26/59/2008/.

Kudeki, E. and Milla. M.: Incoherent scatter spectrum theory for modes propagating perpendicular to the geomagnetic field, J. Geophys. Res., 111, A06306, doi:10.1029/2005JA011546, 2006.

Kudeki, E., Bhattacharyya, S., and Woodman, R. F., A new approach in incoherent scatter $F$ region $\boldsymbol{E} \times \boldsymbol{B}$ drift measurements at Jicamarca, J. Geophys. Res., 104, 28145-28162, 1999.

Lawson, C. W. and Hanson, R. J.: Solving Least Squares Problems, SIAM, Philadelphia, 1987.

Lehtinen, M. S.: Statistical theory of incoherent scatter radar measurements, Tech. Rep. 86/45, Eur. Incoherent Scatter Sci. Assoc., Kiruna, Sweden, 1986.

Lehtinen, M. S. and Huuskonen, A.: General incoherent scatter analysis and GUISDAP, J. Atmos. Terr. Phys., 58, 435-452, 1996.

Lehtinen, M. S., Huuskonen, A., and Pirttilä, J.: First experiences of full-profile analysis with GUISDAP, Ann. Geophys., 14, 14871495, 1996, http://www.ann-geophys.net/14/1487/1996/.

Menke, W.: Geophysical Data Analysis: Discrete Inverse Theory, Academic, New York, 1984.

Milla, M. and Kudeki, E.: F-region electron density and $T_{e} / T_{i}$ measurements using incoherent scatter power data collected at ALTAIR, Ann. Geophys., 24, 1333-1342, 2006, http://www.ann-geophys.net/24/1333/2006/.

Nikoukar, R., Kamalabadi, F., Kudeki, E., and Sulzer, M.: An efficient near-optimal approach to incoherent scat- 
ter radar parameter estimation, Radio Sci., 43, RS5007, doi:10.1029/2007RS003724, 2008.

Nygrén, T.: Introduction to incherent scatter measurements, Invers Publications, Sodankyla, Finland, 1996.

Pingree, J. E.: Incoherent scatter measurements and inferred energy fluxes in the equarotial $F$-region ionosphere, $\mathrm{PhD}$ thesis, Cornell Univ., Ithaca, NY, 1990.

Rodrigues, F. S., Nicolls, M. J., and Hysell, D. L.: Improved electron density measurements at Jicamarca, J. Geophys. Res., 112, A11315, doi:10.1029/2007JA012598, 2007.

Scherliess, L. and Fejer, B. G.: Radar and satellite global equatorial $F$ region vertical drift model, J. Geophys. Res., 105, 6829-6842, 1999.

Schunk, R. W. and Nagy. A. F.: Ionospheres - Physics, Plasma Physics, and Chemistry, Cambridge Univ. Press, 2000.

Sheffield, J.: Plasma Scattering of Electromagnetic Radiation, Academic, New York, 1975.

Sulzer, M. P.: A radar technique for high range resolution incoherent scatter autocorrelation function measurements utilizing the full average power of klystron radars, Radio Sci., 21, 1033-1040, 1986.
Sulzer, M. P. and Gonzalez, S.: The effect of electron Coulomb collisions on the incoherent scatter spectrum in the $F$ region at Jicamarca, J. Geophys. Res., 104, 22535-22552, 1999.

Tarantola, A.: Inverse Theory, Elsevier, New York, 1987.

Turunen, T.: GEN-SYSTEM - new experimental philosophy for EISCAT radar, J. Atmos. Terr. Phys., 48, 777-785, 1986.

Virtanen, I. I., Lehtinen, M. S., Nygrén, T., Orispää, M., and Vierinen, J.: Lag profile inversion method for EISCAT data analysis, Ann. Geophys., 26, 571-581, 2008, http://www.ann-geophys.net/26/571/2008/.

Woodman, R. F.: A general statistical instrument theory of atmospheric and ionospheric radars, J. Geophys. Res., 96, 7911-7915, 1991.

Woodman, R. F.: On a proper electron collision frequency for a Fokker-Planck collision model with Jicamarca applications, J. Atmos. Sol. Terr. Phys., 66.17, 1521-1541, 2004.

Woodman, R. F. and Hagfors, T.: Methods for the measurement of vertical ionospheric motions near the magnetic equator by incoherent scattering, J. Geophys. Res., 74, 1205-1212, 1969.

Woodman, R. F. and La Hoz, C.: Radar observations of $F$ region equatorial irregularities, J. Geophys. Res., 81, 5447-5466, 1976. 